\title{
The Extension of the Massless Fermion Behaviour in the Cosmic String Spacetime
}

\author{
Bengü Çağatay, ${ }^{1}$ Özlem Yeşiltaş ${ }^{(D},{ }^{2}$ and Anıl L. Aygüin ${ }^{2}$ \\ ${ }^{1}$ Turkish Atomic Energy Authority, Sarayköy Nuclear Research and Training Centre, 06983 Ankara, Turkey \\ ${ }^{2}$ Gazi University, Faculty of Science, Physics Department, 06500 Teknikokullar/Ankara, Turkey \\ Correspondence should be addressed to Özlem Yeşiltaş; yesiltas@gazi.edu.tr
}

Received 25 April 2020; Revised 10 August 2020; Accepted 13 August 2020; Published 3 September 2020

Academic Editor: Burak Bilki

Copyright (C) 2020 Bengü Çăgatay et al. This is an open access article distributed under the Creative Commons Attribution License, which permits unrestricted use, distribution, and reproduction in any medium, provided the original work is properly cited. The publication of this article was funded by $\mathrm{SCOAP}^{3}$.

In this work, we have obtained the solutions of a massless fermion which is under the external magnetic field around a cosmic string for specific three potential models using supersymmetric quantum mechanics. The constant magnetic field, energy-dependent potentials, and position-dependent mass models are investigated for the Dirac Hamiltonians, and an extension of these three potential models and their solutions is also obtained. The energy spectrum and potential graphs for each case are discussed for the $\alpha$ deficit angle.

\section{Introduction}

The massless Dirac character of the low energy electrons moving has attracted much interest in physics due to the graphene's important electronic properties [1]. There are a series of studies on the interaction of graphene electrons in perpendicular magnetic fields which have been carried out in order to find a way for confining the charges [2,3]. Graphene and its derivatives (nanotubes, fullerenes) have become a mine of novel technologies and studying various aspects of physics through its many subfields as well as cosmological models on honeycomb branes [4]. Moreover, topological defects which are disorders lead to important effects on the electronic properties of low dimensional systems. A cosmic string and topological defect relationship is studied where the topological defects are located at arbitrary positions on the graphene plane [5]. In line with the efforts to combine general relativity theory with quantum mechanics, these studies are of great interest [6-8]. Cosmic strings were introduced by Kibble in 1976 [9], and the geometry of the massless cosmic strings is examined in the large scale limit of the model given in [10]. In relativistic quantum mechanics, Lie algebraic approaches [11], a hydrogen atom in the background of an infinitely thin cosmic string [12], scalar particle dynamics in gravity's rainbow through the space-time of a cosmic string [13], and $N=2$ supersymmetric approach to cosmic string dynamics [14] are some recent studies about the topic. For more details about the technique of SUSY, the readers may look at the book [15]. The origin of supersymmetric (SUSY) quantum mechanics is based on the very early development of quantum mechanics by Dirac who found a method to factorize the harmonic oscillator and Schrödinger who noted symmetries in the solutions to his equation [16]. After then, the context of SUSY QM was first studied by Witten [17] and Cooper and Freedman [18]. This theory, which still attracts much attention today, has also application in optics [19] and biophysics [20]; it has a very important place in both relativistic [21] and nonrelativistic quantum mechanics $[22,23]$. This work points the dynamics of a massless fermion out when it is under the influence of a magnetic field. Using the fundamental aspects of SUSY quantum mechanics, three different potential models are discussed for the different values of a deficit angle. Moreover, supersymmetric extension of these models provides generated unknown and complex potential models. The paper is organized as follows: low dimensional Dirac equation is written in the curved spacetime using cosmic string line element and aspects of SUSY quantum mechanics given in Section 2. 
Section 3 is devoted to all potential models which are radial, hyperbolic, and nonlinear ones, and their solutions are given. Their energy and potential function graphs are shown. Section 4 includes the extended new quantum mechanical potential models and their solutions. Energy and potential graphs are also shown. We conclude our results in Section 5.

\section{Dirac Hamiltonian for a Cosmic String in the Gravitational Background}

A massless fermion dynamics can be represented by the Dirac equation-Weyl equation. Specially, we assume that the particle occurs in the external electromagnetic field in a cosmic string spacetime. Then, the particle is described by

$$
i \sigma^{a} e_{a}^{\mu}\left(\nabla_{\mu}+i e A_{\mu}\right) \psi=0
$$

Here, $\sigma^{a}$ are the Pauli matrices, and $\nabla_{\mu}=\partial_{\mu}+\Gamma_{\mu}$ is the covariant derivative. The cosmic string spacetime is represented by the line element which is

$$
d s^{2}=-d t^{2}+d r^{2}+\alpha^{2} r^{2} d \phi^{2}+d z^{2}
$$

where $-\infty<(t, z)<\infty, r \geq 0$, and $0 \leq \phi \leq 2 \pi$. The parameter $\alpha=1-\left(4 \tilde{m} / c^{2}\right)$ is the angular deficit changing in the interval $(0,1]$, where $\tilde{m}$ is the linear mass density of the cosmic string. Here, $e_{\mu}^{a}$ are called as the tetrad fields which connect the Riemannian metric tensor to the flat spacetime metric tensor as

$$
g^{\mu \nu}=e_{a}^{\mu} e_{b}^{v} \eta^{a b}
$$

The tetrads and spinor connections are given, respectively, as

$$
e_{\mu}^{a}(x)=\left(\begin{array}{cccc}
1 & 0 & 0 & 0 \\
0 & \cos \phi & -\alpha r \sin \phi & 0 \\
0 & \sin \phi & \alpha r \cos \phi & 0 \\
0 & 0 & 0 & 1
\end{array}\right),
$$

where

$$
\Sigma^{a b}=\frac{i}{2}\left[\gamma^{a}, \gamma^{b}\right]
$$

where $\gamma^{a}$ is the standard Dirac matrices defined in the Minkowski spacetime:

$$
\gamma^{0}=\beta=\left(\begin{array}{cc}
1 & 0 \\
0 & -1
\end{array}\right)
$$

$$
\begin{gathered}
\gamma^{i}=\beta \alpha^{i}=\left(\begin{array}{cc}
0 & \sigma^{i} \\
-\sigma^{i} & 0
\end{array}\right), \\
\Sigma^{i}=\left(\begin{array}{cc}
\sigma^{i} & 0 \\
0 & \sigma^{i}
\end{array}\right) .
\end{gathered}
$$

We use the units $\hbar=c=1$; the line element of the stationary cosmic string spacetime is written as

$$
d s^{2}=-d t^{2}+d r^{2}+\alpha^{2} r^{2} d \phi^{2}+d z^{2}
$$

with $-\infty<(t, z)<\infty$ and $r \geq 0$ and $0 \leq \phi \leq 2 \pi$. Here, the parameter $\alpha$ is the angular deficit changing in the interval ( $0,1]$. Considering the components of the metric tensor $g_{\mu \nu}$,

$$
\begin{gathered}
g_{11}=-1, \\
g_{22}=1 \\
g_{33}=\alpha^{2} r^{2} \\
g_{33}=1,
\end{gathered}
$$

and the Christoffel symbols

$$
\Gamma_{i j}^{\mu}=\frac{1}{2} g^{\mu k}\left(\frac{\partial g_{i k}}{\partial q^{j}}+\frac{\partial g_{j k}}{\partial q^{i}}-\frac{\partial g_{i j}}{\partial q^{k}}\right),
$$

the spin connection components can be calculated using the tetrad components and the Christoffel symbols,

$$
\omega_{\mu b}^{a}=\eta_{a c} e_{v}^{c} e_{b}^{\sigma} \Gamma_{\sigma \mu}^{v}-\eta_{a c} e_{b}^{v} \partial_{\mu} e_{v}^{c}
$$

which leads to

$$
\omega_{\phi a b}=\left(\begin{array}{cccc}
0 & 0 & 0 & 0 \\
0 & 0 & 1-\alpha & 0 \\
0 & -(1-\alpha) & 0 & 0 \\
0 & 0 & 0 & 0
\end{array}\right) .
$$
[24]

Substituting (17), (7), (11), and (4) in (1), we can obtain

$$
\begin{aligned}
& i \gamma^{0} \frac{\partial \Psi}{\partial t}+i \gamma^{1}\left(\cos \phi \frac{\partial}{\partial r}-\frac{\sin \phi}{\alpha r} \frac{\partial}{\partial \phi}+\frac{i \sin \phi}{\alpha r} \frac{\omega_{\phi 2}^{1}}{2} \gamma^{3}-\frac{i \sin \phi}{\alpha r} e A_{\phi}\right) \Psi \\
& +i \gamma^{2}\left(\sin \phi \frac{\partial}{\partial r}+\frac{\cos \phi}{\alpha r} \frac{\partial}{\partial \phi}-\frac{i \cos \phi}{\alpha r} \frac{\omega_{\phi 2}^{1}}{2} \gamma^{3}+\frac{i \cos \phi}{\alpha r} e A_{\phi}\right) \Psi \\
& \quad+i \gamma^{3} \frac{\partial \Psi}{\partial z}=0 .
\end{aligned}
$$


Let us use the following form for the spinor as

$$
\psi=\exp (-i E t+i m \phi+i k z)\left(\begin{array}{c}
\varphi_{1}(r) \\
\varphi_{2}(r)
\end{array}\right)
$$

Putting (19) in (18) gives

$$
\begin{aligned}
& \left(\begin{array}{cc}
E-k & i e^{-i \phi}\left(\frac{\partial}{\partial r}+\frac{m}{\alpha r}+\frac{1-\alpha}{2 \alpha r}+\frac{e}{\alpha} \frac{A_{\phi}(r)}{r}\right) \\
i e^{i \phi}\left(\frac{\partial}{\partial r}-\frac{m}{\alpha r}+\frac{1-\alpha}{2 \alpha r}-\frac{e}{\alpha} \frac{A_{\phi}(r)}{r}\right) & E-k
\end{array}\right) \\
& \cdot\left(\begin{array}{l}
\varphi_{1}(r) \\
\varphi_{2}(r)
\end{array}\right)=0 .
\end{aligned}
$$

Then, we get a couple of differential equations:

$$
\begin{gathered}
\left(\frac{d^{2}}{d r^{2}}-\frac{\alpha-1}{\alpha r} \frac{d}{d r}+E^{2}-k^{2}-\frac{(1+2 m-3 \alpha)(-1+2 m+\alpha)}{4 \alpha^{2} r^{2}}\right. \\
\left.+\frac{e(\alpha-2 m) A_{\phi}(r)}{r^{2} \alpha^{2}}-\frac{e^{2} A_{\phi}^{2}(r)}{r^{2} \alpha^{2}}-\frac{e A_{\phi}^{\prime}(r)}{\alpha r}\right) \varphi_{1}(r)=0 \\
\left(\frac{d^{2}}{d r^{2}}-\frac{\alpha-1}{\alpha r} \frac{d}{d r}+E^{2}-k^{2}-\frac{(1+2 m-\alpha)(1-2 m-3 \alpha)}{4 \alpha^{2} r^{2}}\right. \\
\left.-\frac{e(\alpha+2 m) A_{\phi}(r)}{r^{2} \alpha^{2}}-\frac{e^{2} A_{\phi}^{2}(r)}{r^{2} \alpha^{2}}+\frac{e A_{\phi}^{\prime}(r)}{\alpha r}\right) \varphi_{2}=0
\end{gathered}
$$

Next, we transform the system given above into the form

$$
\begin{gathered}
\left(-\frac{d^{2}}{d r^{2}}+W(r)^{2}+W^{\prime}(r)\right) \chi_{1}(r)=\varepsilon \chi_{1}(r), \\
\left(-\frac{d^{2}}{d r^{2}}+W(r)^{2}-W^{\prime}(r)\right) \chi_{2}(r)=\varepsilon \chi_{2}(r),
\end{gathered}
$$

where $\varepsilon=E^{2}-k^{2}$ and

$$
\begin{aligned}
\chi_{1,2}(r) & =r^{1 / 2(1 / \alpha-1)} \varphi_{1,2}(r), \\
W(r) & =\frac{e A_{\phi}(r)+m}{\alpha r}
\end{aligned}
$$

are used. Here, (21) are transformed into (23) with the same energy which also shows that the system is supersymmetric. Let us call two effective Hamiltonians for (23) as $H_{1}$ and $\mathrm{H}_{2}$, respectively:

$$
\begin{aligned}
& H_{1}=-\frac{d^{2}}{d r^{2}}+V_{1}(r)=\mathscr{L}^{-} \mathscr{L}^{+}, \\
& H_{2}=-\frac{d^{2}}{d r^{2}}+V_{2}(r)=\mathscr{L}^{+} \mathscr{L}^{-},
\end{aligned}
$$

and the intertwining operators are defined as

$$
\mathscr{L}^{ \pm}=\mp \frac{d}{d r}+W(r) .
$$

It is noted that (27) can be used to intertwine the system as [25]

$$
H_{1} \mathscr{L}^{-}=\mathscr{L}^{-} H_{2}, H_{2} \mathscr{L}^{+}=\mathscr{L}^{+} H_{1}
$$

Furthermore, one can observe that

$$
\mathscr{L}^{-} \chi_{2}=\varepsilon \chi_{1}, \mathscr{L}^{+} \chi_{1}=\varepsilon \chi_{2}
$$

Let us discuss the exactly solvable potential models for $H_{1}$ and $\mathrm{H}_{2}$ in the next section.

\section{Potential Models}

Now we can arrange some different $A_{\phi}(r)$ vector potential models which give rise to effective Hamiltonians. We can argue that the discrete spectrum of the Hamiltonian $\mathrm{H}_{2}$ is $\varepsilon_{2, n}$ and $\mathscr{L}^{-} \chi_{2,0}=0$; then [26],

$$
\begin{gathered}
\varepsilon_{1, n-1}=\varepsilon_{2, n}, \\
\chi_{1, n-1}(r)=\frac{1}{\sqrt{\varepsilon_{2, n}}} \mathscr{L}^{-} \chi_{2, n}(r), \quad n=1,2, \cdots .
\end{gathered}
$$

3.1. Constant Magnetic Field. The constant magnetic field vector $\vec{B}=\left[0,0, a_{0}\right]$, where $a_{0}$ is a real parameter, is perpendicular to the plane. Then, vector potential component $A_{\phi}($ $r$ ) can be taken as $A_{\phi}(r)=a_{0} r$, and $W(r)$ becomes

$$
W(r)=\frac{e a_{0}}{\alpha}+\frac{m}{\alpha r}
$$

This superpotential is introduced in [15] where the singular potential models are also discussed. Here, we have considered the one-dimensional system. Hence, $V_{1,2}(r)$ functions become

$$
\begin{gathered}
V_{1}(r)=\frac{a_{0}^{2} e^{2}}{\alpha^{2}}+\frac{m(m-\alpha)}{\alpha^{2} r^{2}}+\frac{2 a_{0} e m}{\alpha^{2} r}, \\
V_{2}(r)=\frac{a_{0}^{2} e^{2}}{\alpha^{2}}+\frac{m(m+\alpha)}{\alpha^{2} r^{2}}+\frac{2 a_{0} e m}{\alpha^{2} r} .
\end{gathered}
$$

This system shows that $a_{0}$ should be negative because of the Coulomb's potential; let $a_{0}$ be $a_{0}=-v, v>0$. The solutions of the system (32) which is known as pseudoharmonic potential are already known [27]: 


$$
\begin{gathered}
E_{n}= \pm \sqrt{k^{2}-\frac{v^{2} e^{2}}{\alpha^{2}}-\frac{a_{0}^{2} e^{2} m^{2}}{\alpha^{4}\left(n+(1 / 2)+\sqrt{\left(m(m-\alpha) / \alpha^{2}\right)+(1 / 4)}\right)^{2}}} \\
n=0,1,2, \cdots, \\
X_{1, n}=N_{n} r^{(1 / 2)+\mu} \exp (-\epsilon r)_{1} F_{1}(-n, 2 \mu+1,2 \in r),
\end{gathered}
$$

where $\mu=\left(m(m-\alpha) / \alpha^{2}\right)+(1 / 4), \varepsilon=\left(v^{2} e^{2} / \alpha^{2}\right)-E$, and ${ }_{1} F_{1}$ $(-n, 2 \mu+1,2 \in r)$ are the confluent hypergeometric functions. Let us look at the energy spectrum and the conditions for getting real energy levels. The inside of the square root in (34) must be positive for the real energy spectrum, and

$|k|>\sqrt{\frac{v^{2} e^{2}}{\alpha^{2}}+\frac{a_{0}^{2} e^{2} m^{2}}{\alpha^{4}\left(n+(1 / 2)+\sqrt{\left(m(m-\alpha) / \alpha^{2}\right)+(1 / 4)}\right)^{2}}}, \quad m>1$.

The normalization constant can be written as [27]

$$
N_{n}=\Gamma(2 \mu+1) \sqrt{\frac{n !(2 n+2 \mu+1)}{\Gamma(n+2 \mu+1)}} .
$$

Let us see how the energy and potential functions change with $\alpha$.

As is shown from Figure 1, we can obtain physical potentials for the values of $\alpha$ which takes $0<\alpha<1$. Energy graph in Figure 2 shows that for the values of $\alpha \geq 0.1831$ (green curve), we can get an increasing energy graph. For the greater values of the number $n$, the lower bound for the $\alpha$ increases.

3.2. Potential Models: Energy-Dependent Vector and Scalar Potentials. The energy-dependent potentials are one of the some modified problems of the quantum mechanics [28]. The condition on the density distribution is [28]

$$
\rho_{n}\left(E_{n}, r\right)=\left|\psi_{n}\left(E_{n}, x\right)\right|^{2}\left(1-\frac{\partial V\left(E_{n}, r\right)}{\partial E_{n}}\right) .
$$

As it is seen from the above equation, a positivity condition is

$$
1-\frac{\partial V\left(E_{n}, r\right)}{\partial E_{n}}>0
$$

The details of the related energy-dependent potentials in supersymmetry can be found in [29]. In this case, we take the vector potential $A(r)$ as a complex function

$$
A(r)=\left(C_{1}+S(r)\right) r-\frac{m}{e}
$$

where $C_{1}$ is a constant and $S(r)$ is the complex function. Then, (23) becomes $\chi_{1}^{\prime \prime}(r)+\left(E^{2}-k^{2}-\frac{C_{1}^{2} e^{2}}{\alpha^{2}}-\frac{2 C_{1} e^{2} S(r)}{\alpha^{2}}-\frac{e^{2} S(r)^{2}}{\alpha^{2}}-\frac{e}{\alpha} S^{\prime}(r)\right) \chi_{1}(r)=0$

$$
\chi_{2}^{\prime \prime}(r)+\left(E^{2}-k^{2}-\frac{C_{1}^{2} e^{2}}{\alpha^{2}}-\frac{2 C_{1} e^{2} S(r)}{\alpha^{2}}-\frac{e^{2} S(r)^{2}}{\alpha^{2}}+\frac{e}{\alpha} S^{\prime}(r)\right) \chi_{2}(r)=0,
$$

where we can give the superpotential $W(r)$ as

$$
W(r)=\frac{C_{1} e}{\alpha}+\frac{e S(r)}{\alpha}
$$

and let $S(r)$ be

$$
S(r)=A_{1} \sec h r+B_{1} \tanh r-C_{1},
$$

where $A_{1}, B_{1}, C_{1}$ are the constants. We can get the partner potentials as

$$
\begin{aligned}
V_{1}((r)= & \frac{B_{1}^{2} e^{2}}{\alpha^{2}}+\frac{e\left(A_{1}^{2} e+B_{1}\left(-B_{1} e+\alpha\right)\right)}{\alpha^{2}} \sec h^{2} r \\
& +\frac{A_{1} e\left(2 B_{1} e-\alpha\right)}{\alpha^{2}} \sec h r \tanh r \\
V_{2}(r)= & \frac{B_{1}^{2} e^{2}}{\alpha^{2}}+\frac{e\left(A_{1}^{2} e-B_{1}\left(B_{1} e+\alpha\right)\right)}{\alpha^{2}} \sec h^{2} r \\
& +\frac{A_{1} e\left(2 B_{1} e+\alpha\right)}{\alpha^{2}} \sec h r \tanh r .
\end{aligned}
$$

Here, (45) is known as Scarf II potentials (see [30]). Comparing our system (41), (43), and (44) with the linear energydependence results in [31] can give a solvable model. We can use the parameter $A_{1}$ as energy-dependent potential in our calculations as

$$
A_{1}=\frac{\alpha}{2 e}\left(i-2 \sqrt{E^{2}-\frac{B_{1}^{2} e^{2}}{\alpha^{2}}}\right)
$$

Then, we match our system with the one where linear energy dependency can be found in page 9 in [31] and write the partner potentials as energy-dependent potentials as

$$
\begin{aligned}
V_{1}(r)= & \frac{B_{1}^{2} e^{2}}{\alpha^{2}}+\left(E^{2}+\frac{B_{1} e}{\alpha}-\frac{1}{4}-\frac{2 B_{1}^{2} e^{2}}{\alpha^{2}}-i \sqrt{E^{2}-\frac{B_{1}^{2} e^{2}}{\alpha^{2}}}\right) \sec h^{2} r \\
& +\frac{\left(\alpha-2 B_{1} e\right)}{2 \alpha}\left(-i+2 \sqrt{E^{2}-\frac{B_{1}^{2} e^{2}}{\alpha^{2}}}\right) \sec h r \tanh r \\
V_{2}(r)= & \frac{B_{1}^{2} e^{2}}{\alpha^{2}}+\left(E^{2}-\frac{B_{1} e}{\alpha}-\frac{1}{4}-\frac{2 B_{1}^{2} e^{2}}{\alpha^{2}}-i \sqrt{E^{2}-\frac{B_{1}^{2} e^{2}}{\alpha^{2}}}\right) \sec h^{2} r \\
& +\frac{\left(\alpha+2 B_{1} e\right)}{2 \alpha}\left(i-2 \sqrt{E^{2}-\frac{B_{1}^{2} e^{2}}{\alpha^{2}}}\right) \sec h r \tanh r .
\end{aligned}
$$




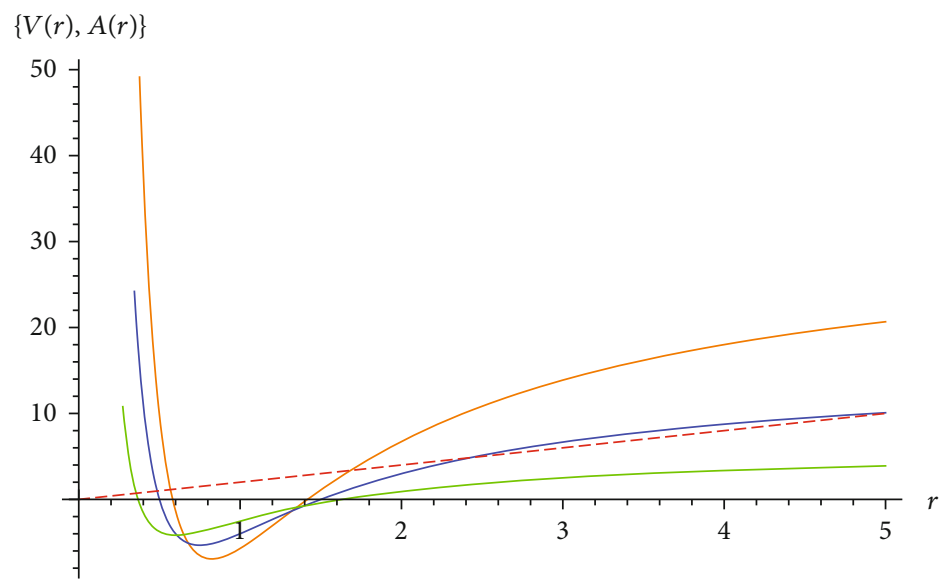

FIGURE 1: The graph of (32) and (40). $V_{1}(r)$ graph versus the position for the different values $\alpha . \alpha=0.5$ for orange, $\alpha=0.5$ for blue, $\alpha=0.8$ for green curves, and the red dashed curve stands for the vector potential.

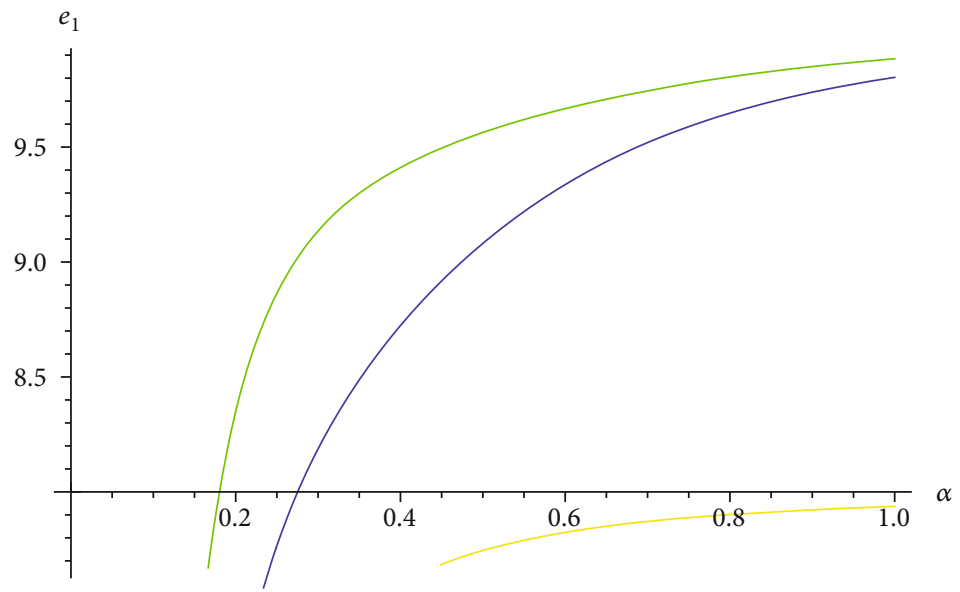

FIGURE 2: The graph of energy eigenvalues with respect to $\alpha$ (34). The green curve is drawn for $k=10, n=1$, and $a_{0}=2$, and the yellow curve is shown for $k=8, n=2$, and $a_{0}=0.2$.

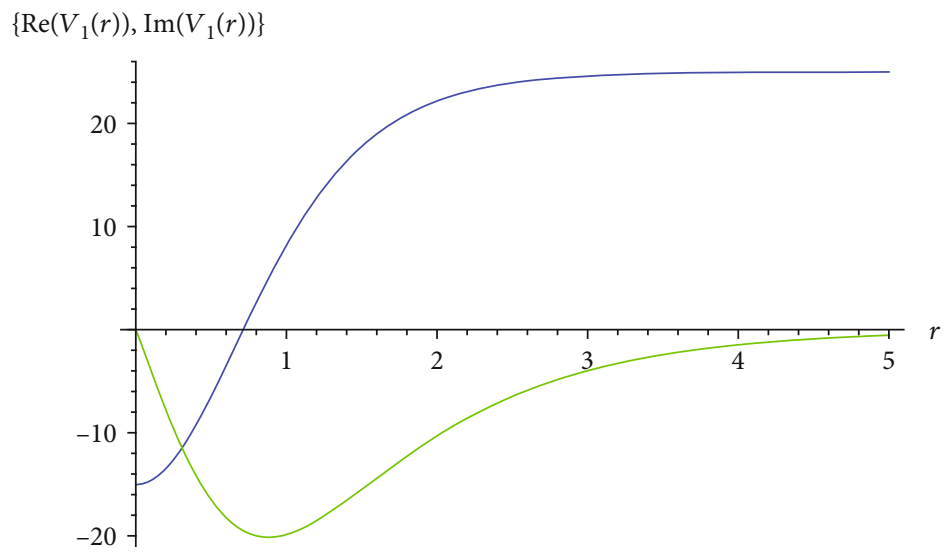

FIGURE 3: The real and imaginary parts of the potential in (48). Green and blue curves are corresponding to the real and imaginary parts, respectively, where $\alpha=0.2$. 


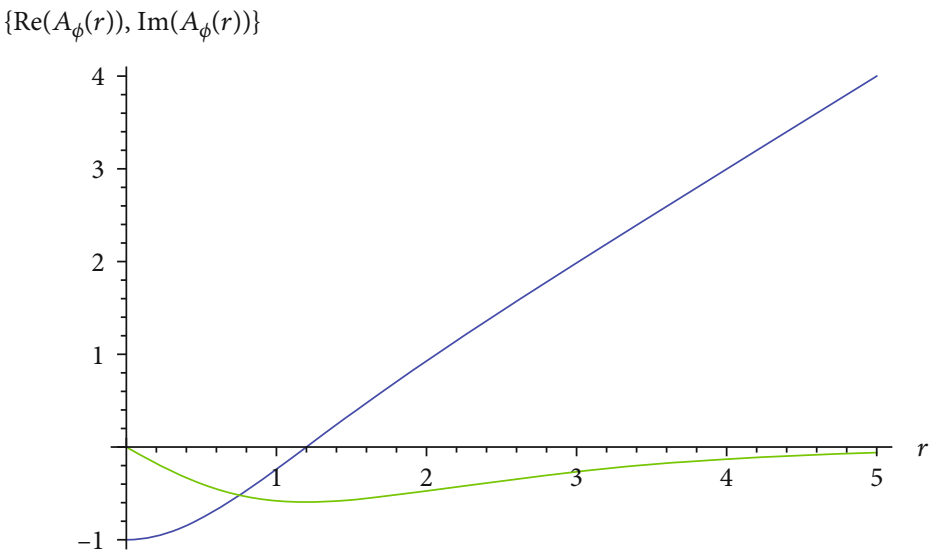

FIgURE 4: The real (green) and imaginary (blue) parts of the vector potential in (40).

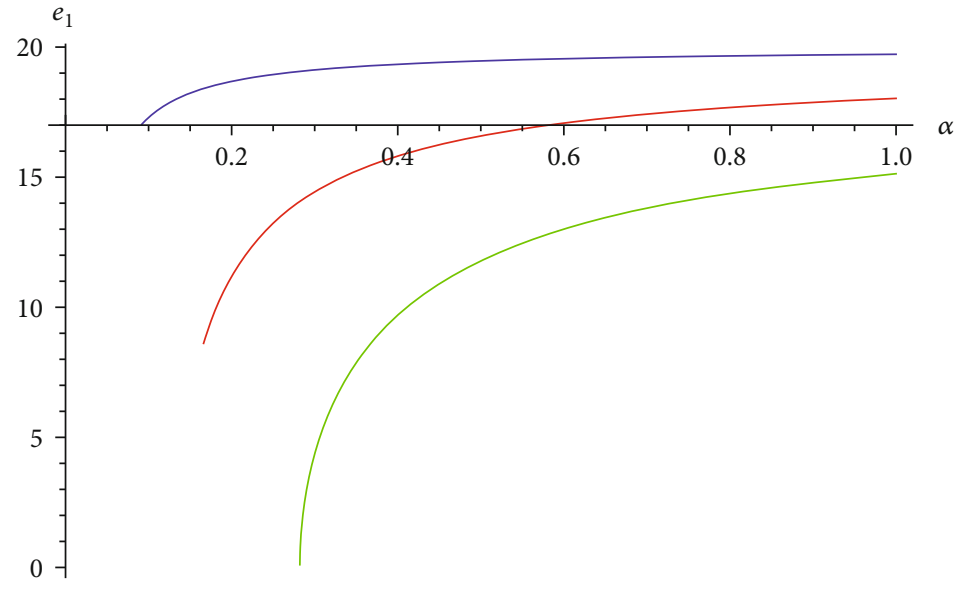

Figure 5: Energy eigenvalues versus the $\alpha$ values for (50).

Then, one can find the energy eigenvalues as

$$
E_{n}= \pm \sqrt{k^{2}-\left(n+\frac{B_{1} e}{\alpha}\right)^{2}+\frac{B_{1}^{2} e^{2}}{\alpha^{2}}}, \quad n=0,1,2, \cdots .
$$

The condition for the real energies is

$$
|k|>\sqrt{\left(n+\frac{B_{1} e}{\alpha}\right)^{2}-\frac{B_{1}^{2} e^{2}}{\alpha^{2}}} .
$$

And the solutions become

$$
\begin{aligned}
\chi_{1, n}= & (1-i \sinh r)^{(1 / 4)-(1 / 2)\left(n+1+\sqrt{k^{2}-\beta^{2}}+4 i \beta\right)} \\
& \cdot(1+i \sinh r)^{(1 / 4)-(1 / 2)\left(n+1+\sqrt{k^{2}-\beta^{2}}-4 i \beta\right)} \\
& P_{n}^{-1 / 2 \sqrt{\left(2+2 n+2 \sqrt{k^{2}-\beta^{2}}+2 i \beta\right)^{2}},-1 / 2 \sqrt{\left(-2 n-2 \sqrt{k^{2}-\beta^{2}}+2 i \beta\right)^{2}}}(i \sinh r) .
\end{aligned}
$$

In Figure 3, we have obtained an effective potential graph for the real potential while we can get a potential barrier from the imaginary part. Figure 4 shows the component of the vector potential whose real component is corresponding to another effective potential curve. In Figure 5, we can see that for the greater values of $n$, the lowest bound for the $\alpha$ again increases as energy increases.

3.3. Potential Models: Position-Dependent Mass Model. The idea of a position-dependent-effective-mass (PDEM) quantum Hamiltonians gains more attention because of the physical applications in graded crystals, quantum dots, and liquid crystals. The hermiticity and the uniqueness of the problem are studied in the region of mathematical physics. For more details, see [32-34]. Let us make a point transformation in (41) given by

$$
r=\int^{z} \frac{d t}{f(t)}
$$


Then, we can get

$$
\begin{aligned}
& f(z)^{2} \chi_{1}^{\prime \prime}(z)+f(z) f^{\prime}(z) \chi_{1}^{\prime}(z) \\
& \quad+\left(E^{2}-k^{2}-\frac{C_{1}^{2} e^{2}}{\alpha^{2}}-\frac{2 C_{1} e^{2} S(z)}{\alpha^{2}}-\frac{e^{2} S(z)^{2}}{\alpha^{2}}+\frac{e}{\alpha} f(z) S^{\prime}(z)\right) \chi_{1}(z)=0 .
\end{aligned}
$$

Let us choose $f(z)=\sqrt{1+\lambda z^{2}}$ and $S(z)=\left(i C_{1} / \sqrt{1+\lambda z^{2}}\right)$ $-c_{1}$; then, (54) turns into

$\left(1+\lambda z^{2}\right) \chi(z)^{\prime \prime}+\lambda z \chi(z)^{\prime}+\left(E^{2}-k^{2}+\frac{C_{1}^{2} e^{2}}{\alpha^{2}\left(1+\lambda z^{2}\right)}+\frac{i C_{1} e \lambda z}{\alpha\left(1+\lambda z^{2}\right)}\right) \chi(z)=0$.

The solutions of the type of equations like (55) are already known. We can write the complete solutions as [35]

$$
\begin{gathered}
E_{n}= \pm \sqrt{k^{2}+n \sqrt{\lambda}\left(\frac{2 C_{1} e-\alpha \sqrt{\lambda}}{\alpha}-n \sqrt{\lambda}\right)} \\
\chi_{n}(z)=N_{n} i^{n}\left(1+\lambda z^{2}\right)^{-s / 2} e^{-r \tan ^{-1}(z \sqrt{\lambda})} P_{n}^{(i r-s-1 / 2,-i r-s-1 / 2)}(i z \sqrt{\lambda}), \\
n=0,1,2, \cdots<s,
\end{gathered}
$$

where

$$
N_{n}=\sqrt{\frac{\sqrt{\lambda} n !(s-n) \Gamma(s-i r-n+1 / 2) \Gamma(s+i r-n+1 / 2)}{\pi 2^{-2 s} \Gamma(2 s-n+1)}} .
$$

$s=\left(2 C_{1} e-\alpha \sqrt{\lambda}\right) / 2 \alpha^{3 / 2}, r=-i / 2$, and $P_{n}^{(a, b)}(y)$ are the corresponding Jacobi polynomials. Now, let us examine the SUSY of this model. First, we need to make a point transformation to (55) which is $\chi_{1}(z)=1 /\left(\left(1+4 \lambda^{2} z^{2}\right)^{1 / 4}\right) Y(z)$. We get

$Y^{\prime \prime}(z)+\left(\frac{1}{4 z^{2}}+\frac{3-4 i A z+4 A^{2} z^{2}}{4 z^{2}\left(1+z^{2} \lambda\right)^{2}}+\frac{-1+i A z+z^{2} \varepsilon^{2}}{z^{2}\left(1+z^{2} \lambda\right)}\right) Y(z)=0$

where $A=C_{1} e / \alpha$. We propose a super potential which is given by

$$
W(z)=\frac{C_{2} z^{2}}{1+z^{2} \lambda}+C_{3}
$$

Then, for (59), the unknown parameters of the superpotential can be obtained as

$$
\begin{aligned}
C_{1} & =-\sqrt{\frac{3}{5} \frac{2 i \alpha \varepsilon}{e},} \\
C_{2} & =4 \sqrt{\frac{3}{5} \varepsilon^{3},} \\
C_{3} & =\frac{3}{5} \varepsilon, \\
\lambda & =-4 \varepsilon^{2} .
\end{aligned}
$$

Figure 6 shows that the energy becomes zero when $\alpha$ $=0.5$ and it is decreasing for the greater values of this angular deficit parameter. In Figure 7, the effective potential graph is shown where the potential well becomes more distinct as $\alpha$ increases.

\section{Extended Potential Models}

Using $V_{1}$ functions given in the previous section, we derive more general partner potentials for each model here.

4.1. Extended Potential Models: Constant Magnetic Field. Let us start with the choice of the superpotential. Here, we want to generate more general potential family of the system given in (32). Now, $W(r)$ is

$$
W(r)=\frac{A}{r}+B r+\frac{f(r)}{g(r)},
$$

where $f(r), g(r)$ are unknown functions. Then, partner potentials can be obtained as

$$
\begin{aligned}
V_{1}(r)= & A+\frac{B(1+B)}{r^{2}}+\frac{2 A B}{r}+\frac{f(r)^{2}}{g(r)^{2}}+\frac{2 A f(r)}{g(r)}+\frac{2 B f(r)}{r g(r)} \\
& -\frac{f^{\prime}(r)}{g(r)}+\frac{f(r) g^{\prime}(r)}{g(r)^{2}},
\end{aligned}
$$

$$
\begin{aligned}
V_{2}(r)= & A+\frac{B(-1+B)}{r^{2}}+\frac{2 A B}{r}+\frac{f(r)^{2}}{g(r)^{2}}+\frac{2 A f(r)}{g(r)}+\frac{2 B f(r)}{r g(r)} \\
& +\frac{f^{\prime}(r)}{g(r)}-\frac{f(r) g^{\prime}(r)}{g(r)^{2}} .
\end{aligned}
$$

Here, we match (63) with (32). If we equate the rational terms to zero in (63) as below

$$
\frac{f(r)^{2}}{g(r)^{2}}+\frac{2 A f(r)}{g(r)}+\frac{2 B f(r)}{r g(r)}-\frac{f^{\prime}(r)}{g(r)}+\frac{f(r) g^{\prime}(r)}{g(r)^{2}}=0,
$$

then, we obtain

$$
f(r)=\frac{\exp (2 A r) r^{2 B} g(r)}{C 1+r^{1+2 B} E_{n}(-2 A r)}
$$

where $n=-2 B$ and the exponential integral function is given by 


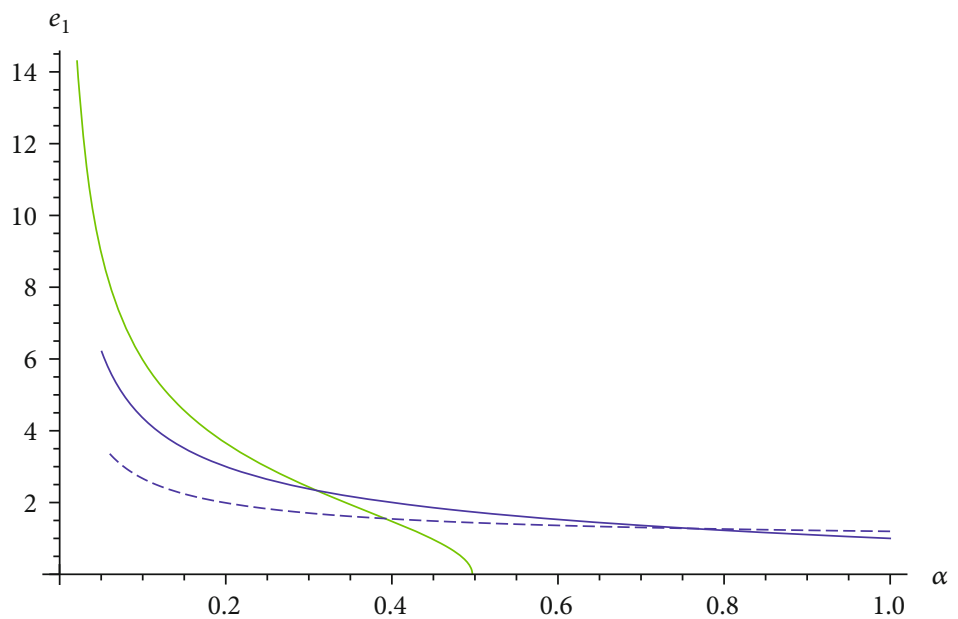

Figure 6: Energy eigenvalues versus $\alpha$ for (56). $k=1, C_{1}=1, n=1$, and $\lambda=5$ for the green curve; $k=1, C_{1}=1, n=1$, and $\lambda=1$ for the red curve; and $k=1, C_{1}=1, n=1$, and $\lambda=0.1$ for the dashed curve.

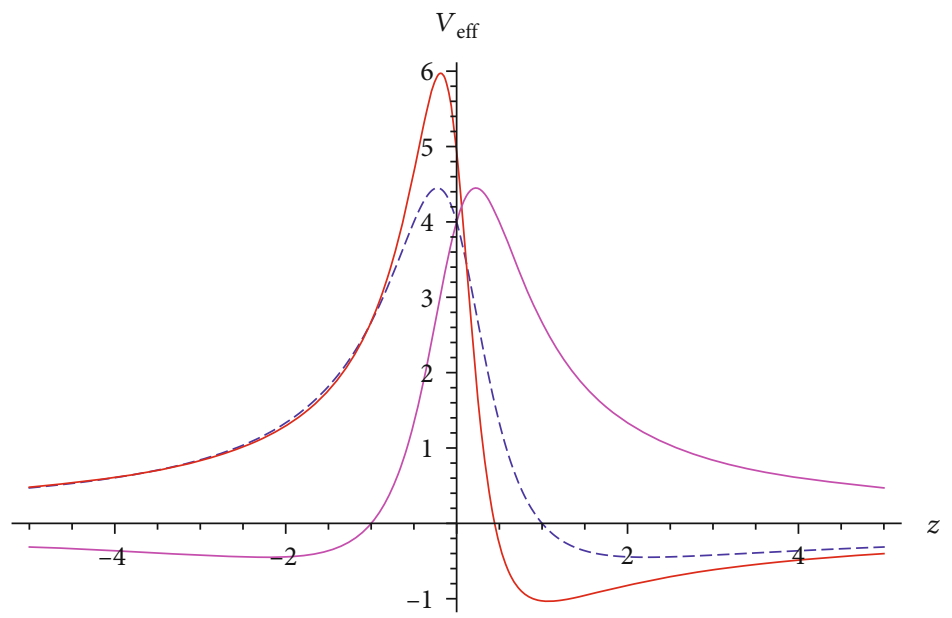

Figure 7: The effective potential graph in (55). $\alpha=0.5$ for the dashed curve, $\alpha=0.9$ for the red curve, and $\alpha=0.1$ for the magenta curve.

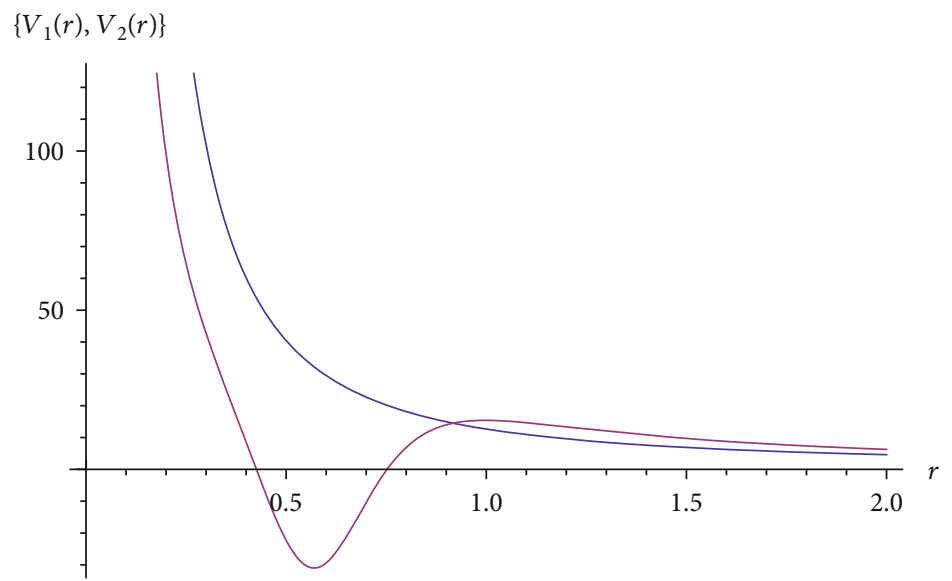

FiguRe 8: $V_{1}(r), V_{2}(r)$ graphs in (68) and (69). $\alpha=0.3$ and $a_{0}=-0.2$. 


$$
E_{n}(z)=\int_{1}^{\infty} \frac{\exp (-r t)}{t^{n}} d t
$$

Then, the partner potentials become

$$
\begin{gathered}
V_{1}(r)=A^{2}+\frac{B(B+1)}{r^{2}}+\frac{2 A B}{r}, \\
V_{2}(r)=A^{2}+\frac{B(B-1)}{r^{2}}+\frac{2 A B}{r} \\
+\frac{2 e^{2 A r} r^{2 B-1}\left(e^{2 A r} r^{1+2 B}+2 C_{1}(B+A r)+2 r^{2 B+1}(B+A r) E_{n}^{-2 A r}\right)}{\left(C_{1}+r^{2 B+1} E_{n}^{-2 A r}\right)^{2}} .
\end{gathered}
$$

Comparing (68) and (32) gives the unknown constants $A$ and $B$ as

$$
\begin{gathered}
A=-\frac{a_{0} e}{\alpha}, \\
B=\frac{-m}{\alpha} .
\end{gathered}
$$

Then, one can find the solution of (69) which is $\chi_{2, n}$ as

$$
\begin{aligned}
X_{2, n}= & \frac{e^{-a_{2} r_{r} a_{1}}}{\varepsilon}\left(c_{2} n_{1} F_{1}\left(1-n, 1+c_{1}, c_{2} r\right)\right) \\
& +\left(A+\frac{B-a_{1}}{r}+a_{2}+\frac{e^{2 A r_{r} 2 B}}{C_{1}+r^{2 B+1} E_{-2 B}(-2 A R)}{ }_{1} F_{1}\left(-n, c_{1}, c_{2} r\right)\right),
\end{aligned}
$$

where

$$
\begin{aligned}
& a_{1}=\frac{1}{2}+\mu, \mu=\frac{m(m-\alpha 1)}{\alpha^{2}}, \\
& a_{2}=\varepsilon, c_{1}=2 \mu+1, c_{2}=2 \varepsilon .
\end{aligned}
$$

We note that (69) shares the same energy level given by (34). In Figure 8, one can see that $V_{2}(r)$ fits an effective potential with a well which is the partner of a Coulombic potential.

4.2. Extended Potential Models: Extended Energy-Dependent Vector and Scalar Potentials. Let us choose the superpotential $W(r)$ as

$$
W(r)=\frac{e\left(a_{1}+i b_{1}\right) \sec h r}{\alpha}+c_{1} f(r)
$$

So, partner potentials are obtained as

$$
\begin{aligned}
V_{1}(r)= & c_{1}^{2} f(r)^{2}-c_{1} f^{\prime}(r)+\frac{2\left(a_{1}+i b_{1}\right) c_{1} e f(r)}{\alpha} \sec h r \\
& +\frac{\left(a_{1}+i b_{1}\right)^{2} e^{2} \sec h^{2} r}{\alpha^{2}}+\frac{\left(a_{1}+i b_{1}\right) e \sec h r \tanh r}{\alpha}, \\
V_{2}(r)= & c_{1}^{2} f(r)^{2}+c_{1} f^{\prime}(r)+\frac{2\left(a_{1}+i b_{1}\right) c_{1} e f(r)}{\alpha} \sec h r \\
& +\frac{\left(a_{1}+i b_{1}\right)^{2} e^{2} \sec h^{2} r}{\alpha^{2}}-\frac{\left(a_{1}+i b_{1}\right) e \sec h r \tanh r}{\alpha} .
\end{aligned}
$$
below

In (73), we can equate the terms to zero which are given

$$
c_{1}^{2} f(r)^{2}-c_{1} f^{\prime}(r)+\frac{2\left(a_{1}+i b_{1}\right) c_{1} e f(r)}{\alpha} \sec h r=0 .
$$

Then, we find

$f(r)=\frac{\exp \left(\left(4 e\left(a_{1}+i b_{1}\right) \tan ^{-1}(\tanh (r / 2))\right) / \alpha\right)\left(2 a_{1} e+2 i b_{1} e+i \alpha\right)}{2 a_{1} e+2 i b_{1} e+i \alpha-2 c_{1} \alpha \exp \left(\left(2\left(2 a_{1} e+2 i b_{1} e+i \alpha\right) \tan ^{-1}(\tanh (r / 2))\right) / \alpha\right)_{2} F_{1}\left(\left(-2 i a_{1} e+2 b_{1} e+\alpha\right) / 2 \alpha, 1,(3 / 2)+\left(\left(b_{1} e-i a_{1} e\right) / \alpha\right),-e^{4 i} \tan ^{-1}(\tanh (r / 2))\right)}$.

Once can give the first partner potential as

$$
V_{1}(r)=\left(\frac{\left(a_{1}+i b_{1}\right) e}{\alpha}\right)^{2} \sec h^{2} r+\frac{\left(a_{1}+i b_{1}\right) e}{\alpha} \sec h r \tanh r
$$

and comparing the coefficients of the hyperbolic functions in (74) with those in (48) gives us

$$
\begin{aligned}
& b_{1}=i\left(a_{1}-A_{1}\right), \\
& A_{1}=\frac{\alpha}{2 e}\left(i-2 \sqrt{E^{2}-1}\right), \\
& B_{1}=\frac{\alpha}{e} .
\end{aligned}
$$

$$
\begin{aligned}
V_{2}(r)= & \left(\frac{\bar{a} e}{\alpha}\right)^{2} \sec h^{2} r-\frac{\bar{a} e \sec h r \tan r}{\alpha} \\
& -\frac{c_{1} e^{\left(4 \bar{a} e \tan ^{-1}(\tanh (r / 2))\right) / \alpha}\left(2 a_{1} e+\left(2 b_{1} e+\alpha\right)\right)\left(\left(2 a_{1} e+i\left(2 b_{1} e+\alpha\right)\right) h(r)\right) \sec h r}{\left(1+e^{4 i \tan ^{-1}(\tanh (r / 2))} \alpha\right)\left(C_{1}\left(2 i a_{1} e+\alpha\right)+2 i \alpha c_{1} e^{(2(2 \bar{a}+i \alpha)) / \alpha}{ }_{2} F_{1}\left(\left(-2 i a_{1} e+2 b_{1} e+\alpha\right) / 2 \alpha, 1,(3 / 2)+\left(\left(e\left(-i a_{1}+b_{1}\right)\right) / \alpha\right),-e^{4 i} \tan ^{-1}\left(\tanh _{(r / 2))}\right)\right)^{2}\right.},
\end{aligned}
$$




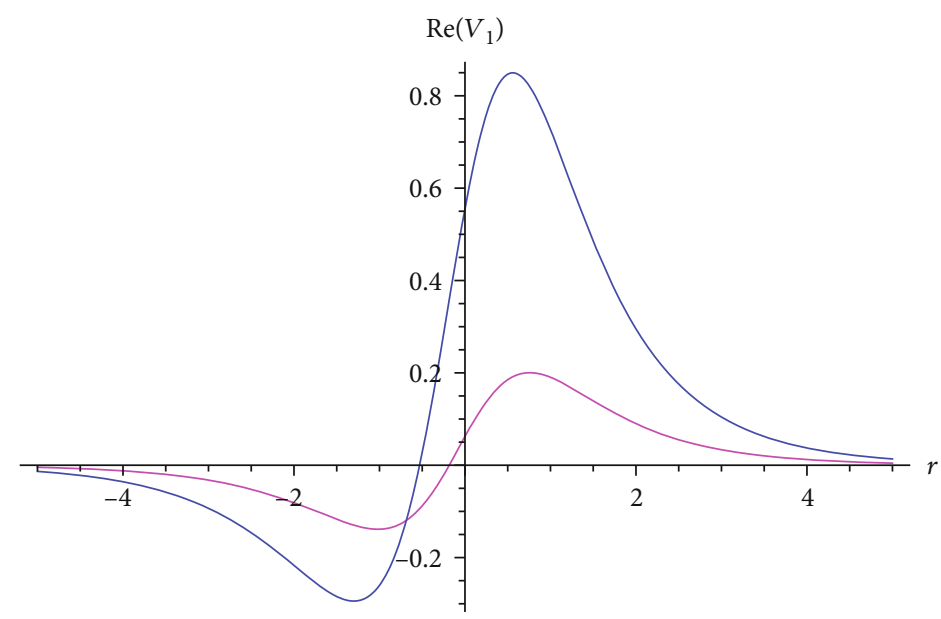

FIGURE 9: $V_{1}(r)$ graph in (78). $\alpha=0.3$ for the blue curve and $\alpha=0.9$ for the magenta curves. $a_{1}=0.3$ and $b_{1}=0.2$.

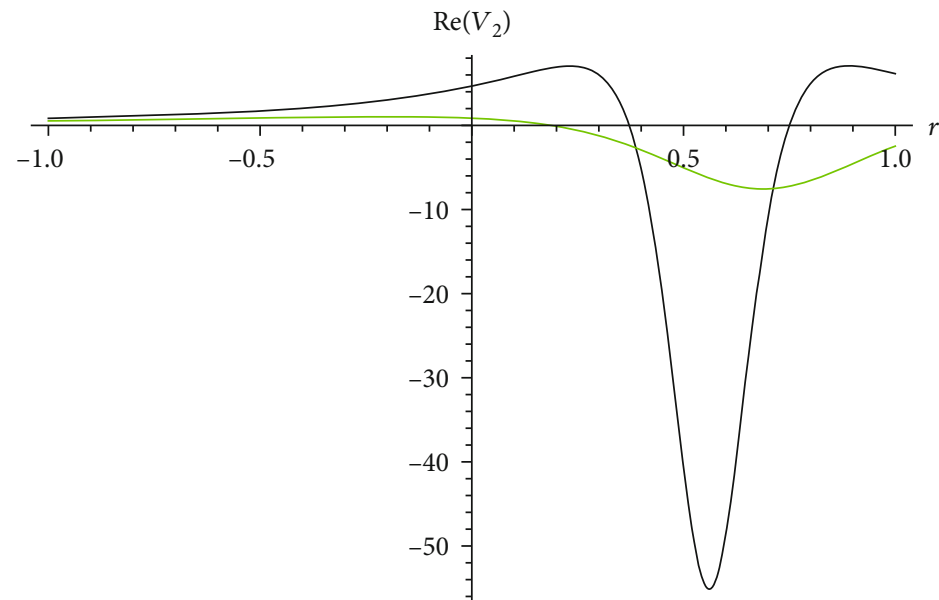

Figure 10: $V_{2}(r)$ in (80), $\alpha=0.3$ for the black curve and $\alpha=0.9$ for the green curve. $a_{1}=0.3$ and $b_{1}=0.2$.

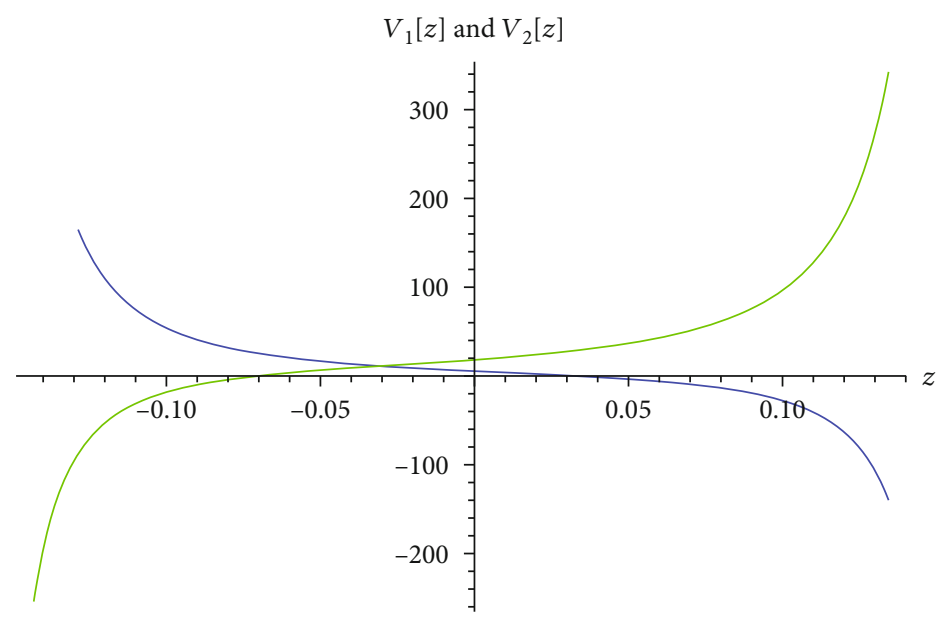

Figure 11: The graph of the partner potentials which are $V_{1}(z)$ in (86) (blue) and $V_{2}(z)$ in (89) (green). 
where

$$
\begin{aligned}
h(r)= & \left(2 C_{1} \alpha e^{\left(2(2 \bar{a} e+2 i \alpha) \tan ^{-1}(\tanh (r / 2))\right) / \alpha}+\left(1+e^{4 i \tan ^{-1}(\tanh (r / 2))}\right)\right. \\
& \left.\cdot\left(4 \bar{a} e C_{1}+\alpha c_{1} e^{\left(\left(4 \bar{a} e \tan ^{-1}(\tanh (r / 2))\right) / \alpha\right)} \cosh r\right)\right) \\
& -8 \bar{a} c_{1}\left(e^{\left(2(2 \bar{a}+3 i \alpha) \tan ^{-1}(\tanh (r / 2))\right) / \alpha}+e^{\left(2(\bar{a}+i \alpha) \tan ^{-1}(\tanh (r / 2))\right) / \alpha}\right) e \alpha_{2} F_{1} \\
& \cdot\left(\frac{-2 i a_{1} e+2 b_{1} e+\alpha}{2 \alpha}, 1, \frac{3}{2}+\frac{e\left(-i a_{1}+b_{1}\right)}{\alpha},-e^{4 i \tan ^{-1}(\tanh (r / 2))}\right)
\end{aligned}
$$

and we use $\bar{a}=a_{1}+i b_{1}$; the constants $\lambda_{1,2}$ are given by

$$
\begin{aligned}
& \lambda_{1}=-\left(1+n+\sqrt{k^{2}-\beta^{2}}+i \beta\right), \\
& \lambda_{2}=-\left(-n-\sqrt{k^{2}-\beta^{2}}+i \beta\right) .
\end{aligned}
$$

One can find a higher potential barrier as $\alpha$ decreases in Figure 9 for the real part of $V_{1}$, and for the real part of the partner potential $V_{2}$, it shows a potential getting deeper as $\alpha$ decreases in Figure 10.

4.3. Extended Potential Models: Extended Position-Dependent Mass Model. The exact solutions of the position-dependent effective mass Schrödinger equation for the nonlinear isotonic oscillator are found in [36]. In this case, we search for a new family of the extension of the nonlinear isotonic oscillator type potentials within the approach mentioned in (55). Now, we take $W(z)$ which has a form

$$
W(z)=\frac{C_{2} z^{2}}{1+\lambda z^{2}}+C_{3}+G(z)
$$

where $G(z)$ is the unknown function which can be found by the following equations:

$$
\begin{aligned}
W(z)^{2} & -W^{\prime}(z)=C_{3}^{2}+\frac{C_{2}^{2} z^{4}+2 C_{2} z^{3} \lambda}{\left(1+z^{2} \lambda\right)^{2}}+\frac{2 C_{2} C_{3} z^{2}-2 C_{2} z}{1+z^{2} \lambda} \\
& +2 C_{3} G_{1}(z)+\frac{2 C_{2} z^{2} G_{1}(z)}{1+z^{2} \lambda}+G_{1}(z)^{2}-G_{1}^{\prime}(z)
\end{aligned}
$$

$$
2 C_{3} G_{1}(z)+\frac{2 C_{2} z^{2} G_{1}(z)}{1+z^{2} \lambda}+G_{1}(z)^{2}-G_{1}{ }^{\prime}(z)=0 .
$$

Here, we note that

$$
V_{1}(z)=\frac{\varepsilon^{2}(3-8 \sqrt{15} \varepsilon z)}{5\left(1-4 \varepsilon^{2} z^{2}\right)^{2}}
$$

From (85), $G(z)$ can be obtained as

$$
G(z)=\frac{2 \varepsilon(10+\sqrt{15})^{\sqrt{3 / 5} \tanh ^{-1}(2 \varepsilon z)}}{-2(10+\sqrt{15}) e^{\sqrt{3 / 5} \tanh ^{-1}(2 \varepsilon z)} \varepsilon z+2(10+\sqrt{15}) \varepsilon C_{1}+h(z)},
$$

where

$$
\begin{aligned}
h(z)= & e^{\sqrt{3 / 5} \tanh ^{-1}(2 \epsilon z)}\left(-(10+\sqrt{15}){ }_{2} F_{1}\left(\frac{\sqrt{3 / 5}}{2}, 1,1+\frac{\sqrt{3 / 5}}{2}\right.\right. \\
& \left.-e^{2 \tanh ^{-1}(2 \epsilon z)}\right)+\sqrt{15} e^{\sqrt{3 / 5} \tanh ^{-1}(2 \in z)}{ }_{2} F_{1} \\
& \left.\cdot\left(\frac{\sqrt{3 / 5}}{2}, 1,2+\frac{\sqrt{3 / 5}}{2},-e^{2 \tanh ^{-1}(2 \epsilon z)}\right)\right) .
\end{aligned}
$$

Now, we can find the $V_{2}(z)$ as

$$
V_{2}(z)=\frac{\varepsilon^{2}}{15}\left(\frac{3(3+8 \sqrt{15} \varepsilon z)}{\left(1-4 \varepsilon^{2} z^{2}\right)^{2}}+c_{1}(z)+c_{2}(z)\right)
$$

where

$$
\begin{gathered}
c_{1}(z)=\frac{600(23+4 \sqrt{15}) e^{2 \sqrt{3 / 5} \tanh ^{-1}(2 \varepsilon z)}}{(10+\sqrt{15})\left(-2 \varepsilon C_{1}+e^{\sqrt{3 / 5} \tanh ^{-1}(2 \varepsilon z)}\left(2 \varepsilon z+{ }_{2} F_{1}(1, \sqrt{3 / 2} / 2,1+(\sqrt{3 / 2} / 2), 1+(2 /(-1+2 \varepsilon z)))\right)\right)-15 e^{\left((10+\sqrt{15}) \tanh ^{-1}(2 \varepsilon z)\right) / 5} \lambda(z)}, \\
c_{2}(z)=\frac{120\left(3+2 \sqrt{15} e^{\sqrt{3 / 5} \tanh ^{-1}(2 \varepsilon z)}\right)}{\left(-1+4 \varepsilon^{2} z^{2}\right)\left((10+\sqrt{15})\left(-2 \varepsilon C_{1}+e^{\sqrt{3 / 5} \tanh ^{-1}(2 \varepsilon z)}\left(2 \varepsilon z+{ }_{2} F_{1}(1, \sqrt{3 / 2} / 2,1+\sqrt{3 / 2} / 2,1+(2 /(-1+2 \varepsilon z)))\right)-\mu(z)\right)\right.} .
\end{gathered}
$$


and $\lambda(z)={ }_{2} F_{1}(1,1+(\sqrt{3 / 5} / 2)+2+(\sqrt{3 / 5} / 2), 1+(2 /(-1+$ $2 \in z)))$ and $\mu(z)=\sqrt{15} e^{\sqrt{3 / 5} \tanh ^{-1}(2 \in z)}{ }_{2} F_{1}(1,1+\sqrt{3 / 2}, 2+$ $\sqrt{3 / 2}, 1+(2 /(-1+2 \in z)))$

Figure 11 shows that the partner potentials $V_{1}(z)$ and $V_{2}(z)$ behave like $V(z)=z^{3}$ which goes to infinity as $z \rightarrow \pm$ $\infty$. We have seen that the potential pictures can be obtained independently from $\alpha$.

\section{Conclusions}

Using the fundamental concepts of SUSY QM, we have obtained physical solutions for the extended constant magnetic field which leads to a Coulomb problem, energydependent hyperbolic potential and nonlinear isotonic potential, which is argued as the position-dependent mass model for a fermion near cosmic string spacetime. It is observed that the restricted values of the angular deficit $\alpha$ give reasonable behaviours of the potentials for our models.

\section{Data Availability}

The [tex, eps] data used to support the findings of this study are available from the corresponding author upon request.

\section{Conflicts of Interest}

The authors declare that they have no conflicts of interest.

\section{References}

[1] K. S. Novoselov, A. K. Geim, S. V. Morozov et al., "Electric Field Effect in Atomically Thin Carbon Films," Science, vol. 306, no. 5696, pp. 666-669, 2004.

[2] M. W. C. Dharma-wardana, "Coulomb interactions of massless Dirac fermions in graphene; pair-distribution functions and exchange-driven spin-polarized phases," Solid State Communications, vol. 140, no. 1, pp. 4-8, 2006.

[3] T. K. Ghosh, "Exact solutions for a Dirac electron in an exponentially decaying magnetic field," Journal of Physics: Condensed Matter, vol. 21, no. 4, article 045505, 2009.

[4] J. Nagamatsu, N. Nakagawa, T. Muranaka, Y. Zenitani, and J. Akimitsu, "Superconductivity at $39 \mathrm{~K}$ in magnesium diboride," Nature, vol. 410, no. 6824, pp. 63-64, 2001.

[5] A. Cortijo and M. A. H. Vozmediano, "Erratum to: "Effects of topological defects and local curvature on the electronic properties of planar graphene" [Nucl. Phys. B 763 (2007) 293308]," Nuclear Physics B, vol. 807, no. 3, pp. 659-660, 2009.

[6] F. Silvestro, R. Fabio, and V. Stefano, "The spectrum of the Schrödinger-Hamiltonian for trapped particles in a cylinder with a topological defect perturbed by two attractive delta interactions," International Journal of Geometric Methods in Modern Physics, vol. 15, no. 8, article 1850135, 2018.

[7] E. R. Bezerra de Mello, F. Moraes, and A. A. Saharian, "Fermionic Casimir densities in a conical space with a circular boundary and magnetic flux," Physical Review D, vol. 85, no. 4, article 045016, 2012.

[8] V. R. Khalilov, "Creation of planar charged fermions in Coulomb and Aharonov-Bohm potentials," The European Physical Journal C, vol. 73, no. 8, article 2548, 2013.
[9] T. W. B. Kibble, "Topology of cosmic domains and strings," Journal of Physics A, vol. 9, no. 8, pp. 1387-1398, 1976.

[10] M. van de Meent, "Geometry of massless cosmic strings," Physical Review D, vol. 87, no. 2, article 025020, 2013.

[11] Ö. Yeşiltaş, "SU $(1,1)$ solutions for the relativistic quantum particle in cosmic string spacetime," The European Physical Journal Plus, vol. 130, no. 7, 2015.

[12] G. A. Marquesde and V. B. Bezerra, "Hydrogen atom in the gravitational fields of topological defects," Physical Review D, vol. 66, no. 10, article 105011, 2002.

[13] L. C. N. Santos, C. E. Mota, C. C. Barros Jr., L. B. Castro, and V. B. Bezerra, "Quantum dynamics of scalar particles in the space-time of a cosmic string in the context of gravity's rainbow," 2019, https://arxiv.org/abs/1912.10923.

[14] Ö. Yeşiltaş, "Second-order confluent supersymmetric approach to the Dirac equation in the cosmic string spacetime," The European Physical Journal Plus, vol. 135, no. 2, 2020.

[15] B. K. Bagchi, Supersymmetry in Quantum and Classical Mechanics, Chapman and Hall/CRC, 2000.

[16] E. Schrödinger, "A Method of Determining QuantumMechanical Eigenvalues and Eigenfunctions," Proceedings of the Royal Irish Academy. Section A: Mathematical and Physical Sciences, vol. 46, pp. 9-16, 1940.

[17] E. Witten, "Dynamical breaking of supersymmetry," Nuclear Physics B, vol. 188, no. 3, pp. 513-554, 1981.

[18] F. Cooper and B. Freedman, "Aspects of supersymmetric quantum mechanics," Annals of Physics, vol. 146, no. 2, pp. 262-288, 1983.

[19] B. Midya, H. Zhao, X. Qiao et al., "Supersymmetric microring laser arrays," Photonics Research, vol. 7, no. 3, p. 363, 2019.

[20] F. Brackx, H. De Schepper, and J. Van der Jeugt, "XXXth International Colloquium on Group Theoretical Methods in Physics (ICGTMP) (Group30)," Journal of Physics: Conference Series, vol. 597, article 011001, 2015.

[21] H. P. Laba and V. M. Tkachuk, "Exact energy spectrum of the generalized Dirac oscillator in an electric field," The European Physical Journal Plus, vol. 133, no. 7, 2018.

[22] M. Castillo-Celeita and D. J. Fernández C, "Dirac electron in graphene with magnetic fields arising from first-order intertwining operators," Journal of Physics A: Mathematical and Theoretical, vol. 53, no. 3, article 035302, 2020.

[23] D. J. Fernández C and B. Roy, "Confluent second-order supersymmetric quantum mechanics and spectral design," Physica Scripta, vol. 95, no. 5, article 055210, 2020.

[24] E. R. B. de Mello, A. A. Saharian, and S. V. Abajyan, "Fermionic vacuum polarization by a flat boundary in cosmic string spacetime," Classical and Quantum Gravity, vol. 30, no. 1, article 015002, 2013.

[25] A. A. Andrianov and F. Cannata, "Nonlinear supersymmetry for spectral design in quantum mechanics," Journal of Physics A: Mathematical and General, vol. 37, no. 43, pp. 1029710321, 2004.

[26] Ş. Kuru, J. Negro, and L. M. Nieto, "Exact analytic solutions for a Dirac electron moving in graphene under magnetic fields," Journal of Physics: Condensed Matter, vol. 21, no. 45, article 455305, 2009.

[27] A. Arda and R. Sever, "Exact solutions of the Schrödinger equation via Laplace transform approach: pseudoharmonic potential and Mie-type potentials," Journal of Mathematical Chemistry, vol. 50, no. 4, pp. 971-980, 2012. 
[28] R. J. Lombard, J. Mareš, and C. Volpe, "Wave equation with energy-dependent potentials for confined systems," Journal of Physics G: Nuclear and Particle Physics, vol. 34, no. 9, pp. 1879-1889, 2007.

[29] R. Yekken, M. Lassaut, and R. J. Lombard, "Applying supersymmetry to energy dependent potentials," Annals of Physics, vol. 338, pp. 195-206, 2013.

[30] B. Bagchi and C. Quesne, "An update on the $\mathscr{P} \mathscr{T}$-symmetric complexified Scarf II potential, spectral singularities and some remarks on the rationally extended supersymmetric partners," Journal of Physics A: Mathematical and Theoretical, vol. 43, no. 30 , article $305301,2010$.

[31] A. Schulze-Halberg and P. Roy, "Bound states of the twodimensional Dirac equation for an energy-dependent hyperbolic Scarf potential," Journal of Mathematical Physics, vol. 58, no. 11, article 113507, 2017.

[32] B. Bagchi, P. Gorain, C. Quesne, and R. Roychoudhury, "A general scheme for the effective-mass Schrödinger equation and the generation of the associated potentials," Modern Physics Letters A, vol. 19, pp. 2765-2775, 2011.

[33] M. Gadella, Ş. Kuru, and J. Negro, "Self-adjoint Hamiltonians with a mass jump: General matching conditions," Physics Letters A, vol. 362, no. 4, pp. 265-268, 2007.

[34] J. F. Cariñena, M. F. Rañada, and M. Santander, "Quantization of Hamiltonian systems with a position dependent mass: Killing vector fields and Noether momenta approach," Journal of Physics A: Mathematical and Theoretical, vol. 50, no. 46, article 465202, 2017.

[35] A. Schulze-Halberg and B. Roy, Generalized quantum nonlinear oscillators: Exact solutions and rational extensions, vol. 57, article 102103, 2016.

[36] R. A. Kraenkel and M. Senthilvelan, "On the solutions of the position-dependent effective mass Schrödinger equation of a nonlinear oscillator related with the isotonic oscillator," Journal of Physics A: Mathematical and Theoretical, vol. 42, no. 41, article 415303, 2009. 